\title{
Evaluating the Impact of Alcohol Control Interventions on Suicide Mortality Rates
}

\author{
Xinyang Feng ${ }^{1}$ and Huan Jiang ${ }^{1,2}$ \\ ${ }^{1}$ Dalla Lana School of Public Health, University of Toronto, Toronto, Canada \\ ${ }^{2}$ Institute for Mental Health Policy Research, Centre for Addiction and Mental Health, Toronto, Canada \\ E-mail: xinyang.feng@mail.utoronto.ca
}

Received May 15, 2020

Accepted for publication June 1, 2020

Published online September 26, 2021

\begin{abstract}
Introduction: Given that the impact of regulatory and public policy initiatives cannot usually be tested through traditional randomized controlled trial designs, well-selected, -designed, and -analyzed natural experiments are the method of choice when examining the effects of such enactments on a variety of outcomes. The classic methodology for such evaluations is interrupted time-series (ITS) analysis, which is considered as one of the quasi-experimental designs that use both pre- and post-policy data without randomization. This study tests the impact of alcohol control interventions implemented in different period of times on suicide mortality rates among people 25-74 years of age using ITS.
\end{abstract}

Methods: We mainly use the generalized additive mixed model (GAMM) to capture trend and seasonality in suicide mortality rates while controlling for unemployment rates, financial crisis during 2007-2008, and legal alcohol consumption records. Given the notable differences in alcohol consumption and suicide mortality between males and females, all analyses are conducted gender-specifically.

Results: The ITS shows that the intervention introduced in 2017 has a significant effect on reducing suicide mortality rates for males between 25 and 74 . Following the implementation of the intervention, suicide mortality rates decreased by $23.8 \%$ (95\% CI: $10.2 \%-35.4 \%)$ on average.

Conclusion: The alcohol control intervention that strictly increased the excise tax on alcohol products has been shown to have a strong impact on reducing suicide mortality rates among male adults 25-74 years of age. ITS analyses are one of the strongest evaluative designs and allow a more detailed assessment of the longitudinal impact of an intervention than may be possible with a randomized control trial. 